\title{
ASSOCIATION BETWEEN PROLONGED MATERNAL SMOKELESS TOBACCO USE AND PREGNANCY OUTCOME
}

\author{
RAHMAN ME ${ }^{1}$, HOSSAIN MM ${ }^{2}$, RAHMAN AKMM ${ }^{3}$, DAS SR ${ }^{4}$
}

\begin{abstract}
:
About one third of the adults in the world including increasing number of women (250 million) daily smoke (22\% in developed world \& 9\% of women in developing world). Approximately $28 \%$ women in Bangladesh use smokeless tobacco (ST). The incidence of adverse pregnancy outcome including spontaneous abortion, stillbirth, preterm delivery and giving birth to low birth weight baby $(L B W)$ are quite high in Bangladesh. Tobacco is a preventable cause of maternal and neonatal mortality. This review article intended to find out any association between prolonged maternal smokeless tobacco use and pregnancy outcome. We reviewed various national \& international research papers, thesis papers and articles about smokeless tobacco use and pregnancy outcome. It can be concluded that prolonged use of smokeless tobacco have significant association and risk with spontaneous abortion, stillbirth, preterm delivery and $L B W$ baby.
\end{abstract}

Kew words: Maternal use of ST, Pregnancy outcome, Smokeless tobacco.

J Dhaka Med Coll. 2012; 21(1) : 109-115.

\section{Introduction:}

Tobacco is being used as smoking tobacco and smokeless tobacco (ST). Smoking tobacco is manufactured as cigarettes, bidis, cigars, kreteks, pipes and sticks. Smokeless tobaccos are: chewing tobacco, gul, moist snuff and dry snuff $^{1}$. About one third of the adults in the world including increasing number of women $(250$ million) daily smokes (22\% in developed world $\& 9 \%$ of women in developing world) ${ }^{1}$.

In Bangladesh commonly used smokeless tobaccos are: shada, jorda and gul. These are taken usually with betel quid, areca nut and lime and use of smokeless tobacco mixed with areca nut is very popular here. In Bangladesh smoking prevalence is $45 \%$ among men and $1.5 \%$ among women where $26 \%$ of men and $28 \%$ of women use smokeless tobacco in chewing form ${ }^{2}$.

Tobacco is a priority health problem worldwide and currently it is the second major cause of death in the world. The World Health Organization estimates that smoking now causes three million deaths annually and within two decades will cause more deaths than any single disease ${ }^{3}$.

History of tobacco: Cultivation of tobacco plant probably dates back 8000 years when two species of the plant, $N$. rustica and $N$. tabacum, were dispersed by American Indians through the Southern and Northern American Continent.

When Colombus landed in the New World in 1492, he was offered golden tobacco leaves as a courtesy. Within 150 years of his finding strange leaves, tobacco was being used around the globe. Its rapid spread through the Europeans ${ }^{1}$ and widespread acceptance characterize the addiction to the plant $N$. tabacum.

\section{Types of Tobacco use:}

Now a day, tobacco is being used in various forms. The predominant use of tobacco can be broadly divided into smoking tobacco and smokeless tobacco.

1. Prof. Md. Ekhlasur Rahman, Professor \& Head, Department of Paediatrics ,Dhaka Medical College, Dhaka.

2. Dr. Mohammad Monir Hossain, Dhaka Medical College, Dhaka

3. Dr. A.K.M Matiur Rahman, Assistant Prof of Paediatrics, Dhaka Medical College, Dhaka.

4. Dr. Shishir Ranjan Das, Assistant Prof, Dept of Neonatology, Dhaka Medical College, Dhaka.

Correspondence : Dr ME Rahman, Professor \& Head, Department of Paediatrics, Dhaka Medical College, Dhaka, Bangladesh. Email: hossain.dmc@gmail.com. 


\section{Smoking Tobacco:}

The most widely prevalent and most studied form of tobacco use globally is cigarette smoking. Tobacco smoking has been in vogue for hundreds of years. With the spread of tobacco to Europe and other parts of the world from the 16 th century tobacco smoking soon gained popularity.

Types of smoking tobacco: Tobacco can be smoked in a wide variety of ways. Various forms of smoking tobacco includes ${ }^{4}$ : Cigarettes, Bidis, Cigers, Cheroots, Chuttas, Dhumti, Pipe, Kreteks, Sticks, Hooklis, Chillum, Hookah, Roll-your-own etc.

Health risks of smoking: Smoking harms nearly every organ of the body, causing many diseases and reducing the health of smoker in general. The common and well established health risks of smoking are summarized below ${ }^{1,4,5,6}$.

Cancers - Lung cancer, oral cavity \& pharyngeal cancer, laryngeal cancer, esophageal cancer, pancreatic cancer, bladder $\&$ kidney cancer, cervical \& endometrial cancer, acute myeloid leukemia.

Cardiovascular diseases - Sub-clinical atherosclerosis, coronary heart disease.

Respiratory diseases - Chronic bronchitis \& emphysema, asthma related symptoms.

Gastro-intestinal diseases - Stomach \& duodenal ulcers.

Male reproduction- Sperm: deformity,infertility, impotence.

Female reproduction - earlier menopause, infertility \& delay in conception.

Pregnancy outcomes - Spontaneous abortion, ectopic pregnancy, abruptio placenta, premature rupture of membranes, preterm birth, stillbirth, small for gestational age.

Eye diseases-Nuclear cataract, macular degeneration.

Bone \& Teeth diseases- Osteoporosis in post menopausal women.

\section{Smokeless Tobacco:}

The term smokeless tobacco (ST) is used to describe tobacco that is consumed without heating or burning at the time of use. The use of tobacco in various smokeless forms is very common in India, Bangladesh, Nepal and Myanmar. The different methods of consumption of ST include chewing, sucking, inhalation and applying tobacco preparation to the teeth and gum $4,7,8$.

\section{Types of smokeless tobacco:}

Paan (betel quid) with tobacco: Paan consists of betel leaf, areca nut and slaked lime. In Bangladesh, sun dried tobacco is known as 'Shada'.

Jorda: Jorda is prepared by cutting tobacco leaves into small pieces and boiling them in water with slaked lime and spices until the water evaporates. It is then dried, and colouring and flavouring agents are added. Jorda may be chewed by itself, with areca nut or in betel quid.

Gul: It is a pyrolysed tobacco product. It is marketed in small tin cans and used as a dentifrice.

Snuff: Snuff consists of finely cut or powdered and flavored tobacco. It is of two types: moist snuff and dry snuff. Moist snuff is held in the mouth between cheek and gum. Dry snuff is inhaled through the nose.

Other forms of smokeless tobacco are: Chewing tobacco, Paan masala, Qiwam(Kiwam), Mainpuri tobacco, Hnatsay (Honey Soaked Tobacco), Mawa, Dohra, Tobacco and slaked lime (khaini), Gutka, Red Tooth Powder, Mishri (masheri or misheri), Creamy snuff, Gudhaku,Tuibur / Hidakphu, Tobacco Water, Gundhaku, Bajjar and Creamy snuff.

Pharmacology of smokeless tobacco: ST contains more than 2500 chemical constituents ${ }^{7}$. Among the chemical constituents nicotine is of particular concern. It is a tertiary amine composed of pyridine and pyrolidine ring. In its non-ionized form, nicotine freely permeates through the membranes, including the buccal mucosa and blood brain barrier. Nicotine is absorbed more slowly from ST than from tobacco smoke but peak venous levels are similar. The systemic absorption of nicotine is greater with the use of chewing tobacco or snuff compared with that from 
smoking cigarettes. Nicotine is extensively metabolized by the liver. The major metabolite is cotinine.

\section{Health risks of smokeless tobacco}

The potential adverse health consequences of ST are: Nicotine dependence, accelerated coronary \& peripheral vascular disease, stroke, hypertension, delayed wound healing, reproductive disorders (LBW, prematurity, spontaneous abortion, stillbirth, increase in male fetal wastage, increase in placental weight), peptic ulcer disease, esophageal reflux, dental carries, oral leukoplakia and oral, oropharyngeal, laryngeal \& esophageal cancers $9,10,11$.

\section{Pathophysiology}

The precise mechanism by which tobacco causes adverse pregnancy outcome is not completely understood. Placental and biochemical factors in pregnant women have been considered, and the influence of chemicals in tobacco has been studied.

Smokeless tobacco and smoking both have been associated with abnormal placental pathology.

ST users are exposed to higher level of nicotine than smokers. The systemic absorption of nicotine per dose is greater with use of chewing tobacco (average $4.5 \mathrm{mg}$ from average dose of $7.9 \mathrm{~g}$ chewed over 30 minutes) or snuff (average $3.6 \mathrm{mg}$ from an average $2.5 \mathrm{~g}$ moist snuff kept in mouth for 30 minutes, compared with that from smoking cigarettes (average $1 \mathrm{mg} /$ cigarette) ${ }^{9}$. Nicotine of ST may cause same adverse pregnancy outcome as smoking.

Nicotine is a strong vasoconstrictor. It constricts the uterine artery and umbilical artery and thus reduces the blood flow to the fetus. It increases maternal blood pressure and heart rate. An increase in fetal heart rate is also seen, which is thought to be caused by fetal hypoxia ${ }^{12}$. Nicotine is also associated with significant decrease of most amino acids in both placental tissue and fetal plasma ${ }^{13}$. Nicotine also alters the peripheral autonomic pathways, leading to increased susceptibility to hypoxia induced brain damage and perinatal mortality ${ }^{14}$.

\section{Pregnancy Outcome}

Pregnancy outcome may be defined as results of conception and ensuing pregnancy, including live birth, stillbirth and abortion. ${ }^{15}$ Normal pregnancy outcome is term normal weight (2500gm to $4000 \mathrm{gm}$ ) newborn without any congenital anomaly.

Adverse pregnancy outcomes may be: AbortionSpontaneous \& induced, stillbirth, preterm birth, LBW baby and IUGR.

\section{Tobacco and Spontaneous Abortion}

It is difficult to evaluate the effects of maternal smoking on miscarriage, primarily because of inherent difficulty in measuring the general population's rate of spontaneous abortion. Many studies were done to measure the relationship between smoking and spontaneous abortion.

Ness RB et al. ${ }^{16}$ studied the association between cocaine \& tobacco use and spontaneous abortion among 970 pregnant adolescents and women. A total of 400 adolescent and women had spontaneous abortion. Among them 34.6\% smoked on the basis of urine cotinine assay. The presence of cotinine in urine was independently associated with an increased risk of spontaneous abortion (odd's ratio 1.8 ; 95 percent confidence interval, $1.3-2.6)$.

Gayle CW et al. ${ }^{17}$ examined the risk of spontaneous abortion from environmental tobacco smoke exposure in a prospective study over 5,000 women in California during 19901991. Among non-smokers there was little association by hours of ETS exposure at home or at work. A moderate association with maternal smoking was observed (adjusted odd ratio for $<5$ cigarettes per day, 1 .3).

Walsh $\mathrm{RA}^{18}$ reviewed many studies and summarized that substantial nonexperimental evidence indicates that smoking causes spontaneous abortion. The relative risk of spontaneous abortion in smokers is increased by over one-third after controlling for confounding factors. The positive association between smoking and spontaneous abortion has been found over $80 \%$ of studies, and the data also convincingly demonstrate a dose- 
response gradient based on daily cigarette consumption during pregnancy.

In Bangladesh Hoque M, Rahman $\mathrm{ME}^{19}$ showed spontaneous abortion was higher in ST user group(odds ratio 2.3 and $\mathrm{p}<0.01$ )and in India Krishnamurty $\mathrm{S} 20$ showed similar association(30\% male foetal wastages due to tobacco use).

\section{Tobacco and stillbirth}

Smoking has repeatedly been associated with stillbirth, and the risk generally increases with the amount smoked.

In Bangladesh Hoque M, Rahman $\mathrm{ME}^{19}$ showed stillbirth was higher in ST user group( odd ratio 2.0 and $\mathrm{p}<0.01$ ) than non ST user group.

Raymond EG et al. ${ }^{21}$ conducted a population based cohort study in Sweden between 1983 and 1989 to examine the effects of advanced maternal age, parity and smoking on risk of stillbirth. A total of $6,38,242$ pregnancies were studied. The study revealed increased stillbirth risk in smokers.

Cnattingius et al. ${ }^{22}$ examined the risk factors for late fetal death and early neonatal mortality in a population based prospective study. All Swedish births between 1983 and 1985, 281808 births were included. The overall rate of late fetal death was 3.5 . About $30 \%$ of the pregnant women were recorded as being smokers. Logistic regression analysis showed significant relative risk for late fetal death and maternal smoking.

Dodds et al. ${ }^{23}$ conducted a case control study in Canada between 1999 and 2001. The study included 105 stillbirth cases and 389 live-birth controls. The study found that smoking during first trimester of pregnancy was associated with increased risk of stillbirth (adjusted hazard ratio 2.4 ; 95\% CI, 1.2-4.9).

A prospective cohort study in Mumbai, India by Gupta PC, Sreevidya $\mathrm{S}^{24}$ found the risk for stillbirth in ST users was 2.6 times than non users.

Krishnamurthy S showed that maternal ST ingestion causes delivery of LBW baby 3.2 times and still birth 3 times than non ingested mothers $^{25}$.

\section{Tobacco and Preterm Birth}

Many studies have reported that smoking is associated with preterm labor. A dose response relationship of cigarette smoking and premature delivery was also documented.

A study done in Bangladesh by Hoque $\mathrm{M}$, Rahman $\mathrm{ME}^{19}$ showed preterm delivery was higher in ST user group( odd ratio 3.1 and $\mathrm{p}<$ 0.01).

England $U$ et $\mathrm{al}^{26}$ examined birth weight, preterm birth and pre-eclampsia in women who delivered singleton live born infants in Sweden from 1999 through 2000. After exclusion 789 snuff users, 11240 smokers and 11,495 nonusers remained. Compared with non-users, preterm delivery was increased in snuff users and smokers (adjusted odd ratio 1.98 and 1.57 respectively

Kallen $\mathrm{K}^{27}$ studied 1,413,811 infants born between 1983 and 1996 in Sweden. The study found significant association between maternal smoking and preterm birth. The odd ratios for maternal smoking (>10cigarettes per day and d" 10 cigarettes per day) and preterm delivery were 1.39 and 1.65 respectively (dose response $\mathrm{P}<0.001$ ).

McDonald AD et al. ${ }^{28}$ analyzed data from an occupational and other factors in pregnancy to assess the effects of cigarette, alcohol and coffee consumption on pregnancy outcome. A total of 51512 pregnancies were studied. Smoking accounted for $11 \%$ of preterm births. Prematurity was higher among smokers than non-smokers and increased with number of cigarette smoked.

Another study done in Bangladesh by Hossain $\mathrm{MA}$, Rahman $\mathrm{ME}^{29}$ found that maternal ST use in pregnancy was significantly associated with preterm delivery and carries a risk of having preterm babies 4.6 times than non-ST users.

The prospective cohort study in India by Gupta $\mathrm{PC}$, Sreevidya $\mathrm{S}^{30}$ found that risk of preterm delivery was 1.4 times higher for women who used ST than non user.

\section{Tobacco and LBW}

The most recognized effect of maternal smoking is the increased risk LBW baby. In addition to 
preterm delivery tobacco, both in smoking and smokeless form, is associated with IUGR.

Krishnomurthy $\mathrm{S}$ and Joshi $\mathrm{S}^{25}$ studied 178 singleton live births to see the effects of maternal smokeless tobacco use in pregnancy. The authors found that maternal use of 'Mishri' tobacco in pregnancy was associated with LBW baby ( 3.2 times more risk of having a LBW baby).

A prospective cohort study done in Mumbai, India by Gupta PC, Sreevidya $\mathrm{S}^{30}$ showed that there was 1.6 times more risk of LBW for women who used ST than non users.

Steyn et al. ${ }^{31}$ found that the mean birth weight for non-tobacco users was $3148 \mathrm{gm}$ and that of the smokers $2982 \mathrm{gm}$, resulting in a significant lower birth of $165 \mathrm{gm}$ for babies of smoking mothers $(P=0.005)$. In contrast women using snuff gave birth to infants with a mean birth weight of $3118 \mathrm{gm}$ which is a non-significant decrease $(p=0.52)$ in their infants birth weight compared with those not using tobacco.

Wang $\mathrm{X}$ et al. ${ }^{32}$ conducted a cohort study including 740 white and Hispanic women between 1986 and 1992. This study examined the relationship of birth outcomes to the timing and intensity of maternal active and passive smoking estimated both from self report and from cotinine concentration in maternal urine during early, middle and late gestation. A significant inverse exposure-response relationship between cotinine concentration in urine and infant's size at birth was demonstrated.

More studies done in Bangladesh 19,33,34 showed there was 4.1 times, 2 times and 2.2 times respectively more risk of LBW for women who was used ST than non users.

Deshmukh JS et al. ${ }^{35}$ studied the prevalence of LBW and its association with maternal factors in India. They found tobacco exposure (both smoking and smokeless tobacco) was significantly associated with LBW baby (odd ratio 3.14).

\section{Prevention:}

Tobacco is a preventable cause of adverse pregnancy outcome. So, we should make all the possible efforts to prevent these tobacco related adverse pregnancy outcomes.
Smoking and Tobacco Product Usage (Control) Act 2005

Bangladesh has enacted a tobacco control law in 2005 in accordance with some of the provisions of WHO Framework Convention on Tobacco Control (FCTC).

According to this law 'Tobacco Products' means any product made from Tobacco which can be inhaled through smoking and shall include bidi, cigarette, cherrot, cigar and mixtures used as pipe.

It is noteworthy that the definition of tobacco products does not include Smokeless Tobacco. So, the act should be revised to control use of smokeless tobacco.

\section{Nicotine Replacement Therapy}

Intervention to quit tobacco includes behavior therapy and pharmacotherapy. The only drug treatment readily available for quitting tobacco use is nicotine replacement therapy. Currently available drugs are Nicotine gum, transdermal nicotine patch, nicotine aerosols, Bupropion SR, Zyban.

\section{Nicotine vaccine:}

Researches for human nicotine vaccine are going on. In near future, it may be possible to use nicotine vaccine to protect the fetus from the detrimental effects of maternal tobacco use.

NicVAX ${ }^{36}$ is a nicotine conjugate vaccine intended to reduce or eliminate physical addiction to nicotine. According to National Institute of Drug Abuse, N1cVAX can potentially be used to inoculate against addiction. This proprietary vaccine is being developed by Nabi Biopharmaceuticals. NicVAX consists of the hapten 3'- aminornethylnicotine which has been conjugated to Pseudomonas aeroginosa exoprotein.

The vaccine is designed to cause the immune system to produce antibodies that bind to nicotine and prevent it from entering the brain. NicVAX is still under development; it is currently on phase III of testing.

\section{Conclusion:}

Public awareness about the adverse pregnancy outcome of tobacco use should be increased, 
using all possible channels of communication of mass education as well as for influencing women. Women \& female children should be discouraged to take and to be habituated to smoking \& ST. Women who are already habituated to smoking or any other tobacco product should be advised to quit this habit before being pregnant and during pregnancy. Women should be helped who are willing to quit tobacco. There should be restriction about the advertising and promotion of tobacco products.

\section{References:}

1. World Health Organization. The Tobacco Atlas. Available from: http://www.who.int/tobacco/ statistics/tobacco_atlas/en/ (accessed on 02.11.2010).

2. Global adult tobacco survey(GATS): Bangladesh.World Health Organization;2009. Availale from:http://www.searo.who.int/Link Files/Regional__ Tobacco_Surveillance System_GATSBAN_FullReport 2009.pdf.

3. Tobacco. West Encyclopedia of American Law (Full article) from Answer.com (accessed on 02.11.2010).

4. Tobacco. From Wikipedia, the free encyclopedia (accessed on 02.11.2010).

5. Reddy KS, Gupta PC. Report on tobacco control in India, New Delhi: Ministry of Health and Welfare, Government of India; 2004.

6. US Department of Health and Human Services. The health consequences of smoking: A report of the surgeon general. Center for Disease Control and Preventoion. CDC, Atlanta, USA. 2004.

7. Tobacco. Chemistry daily. The Chemistry Encyclopedia (accessed on 02.11.2010).

8. Sinha DN. Report on oral tobacco use and its implications in South East Asia. School of Preventive Oncology, Patna. WHO SEARO. 2004.

9. Benowitz NL. Pharmacology of smokeless tobacco use: Nicotine addiction and nicotine-related health consequences. In: Behington W. ed. Nicotine Effect and Addiction. National cancer institute - an international perspective. US Department on Health and Human Services. Public Health Service. 1992; 93: 219-27.

10. Gupta PC, Ray CS. Smokeless tobacco and health in India and South Asia. Respirology 2003; 8: 419-31.

11. Ebbert JO, Carr AB, Dale LC. Smokeless tobacco: an emerging addiction. Med Clin North Am 2004; 88(6): 1593-605.
12. Lambers D, Clark K. The maternal and fetal physiologic effects of nicotine. Semin Perinatol 1996; 20(2): 115-26.

13. Pastrakuljic A, Derewlany LO, Koren G. Maternal cocaine use and cigarette smoking in pregnancy in relation to amino acid transport and fetal growth. Placenta 1999; 20(7): 499-512.

14. Slotkin TA. Fetal Nicotine or cocaine exposure: which one is worse? JPET 1998; 285: 931-45.

15. Definition of pregnancy outcome. Reference.MD. Encyclopedia of medical concept (accessed on 22.12.2009).

16. Ness RB, Grisso JA, Hirschinger N, Markovic N, Shaw LM, Day NL, et al. Cocaine and tobacco use and the risk of spontaneous abortion. N Engl J Med 1999; 340(5): 333-9.

17. George L, Granath F, Johansson AL, Annerén G, Cnattingius S. Environmental tobacco smoke and risk of spontaneous abortion. Epidemiology 2006; 17(5): 500-5.

18. Walsh RA. Effects of maternal smoking on adverse pregnancy outcomes: examination of the criteria of causation. Hum Biol 1994; 66: 1052-92.

19. Hoque M, Rahman ME. Pregnancy outcome of mothers who used smokeless tobacco for prolonged period: a retrospective cohort study. MD Thesis; Shahajalal University of Science \& Technology: Sylhet; 2009.

20. Krishnamurthy S. Maternal tobacco use and adverse reproductive outcome. Nat Med J India 1997; 10(1): 2-4.

21. Raymond EG, Cnattingius S, Liely JL. Effects of maternal age, parity and smoking on the risk of stillbirth. Br J Obstet Gynaecol 1994; 101(4): 3016.

22. Cnattingius S, Haglund B, Merik O. Cigarette smoking as risk factor for fetal death and early neonatal death. BMJ 1998; 297: 258-62.

23. Dodds L, Wing WD, Fell DB, Armson BA, Allen A, Nimrod C. Stillbirth risk factors according to timing of exposure. Ann Epidemiol 2006; 16: 607-13.

24. Gupta PC, Sreevidya S. Smokeless tobacco use and risk of stillbirth : a cohort study in Mumbai, India. Epidemiology 2006 Jan; 17(1): 47-51.

25. Krishnamurthy S, Joshi S. Gender differences and low birth weight with maternal smokeless tobacco use in pregnancy. J Trop Pediatr 1993; 39: 253-4.

26. England LJ, Levine RJ, Mills JL, Klebanoff MA, Yu KF, Cnattingius S. Adverse pregnancy outcome in snuff users. Am J Obstet Gynecol 2003; 189(4): 939-43. 
27. Kallen K. The impact of maternal smoking during pregnancy on delivery outcome. Eur J Public Health 2001; 11: 329-33.

28. McDonald AD, Armstrong BG, Sloan M. Cigarette, alcohol, and coffee consumption and prematurity. Am J Public Health 1992; 82: 87-90.

29. Hossain MA. Role of Maternal smokeless Tobacco ingestion during pregnancy in delivery of preterm babies. [MD Thesis]. Sylhet: Shahajalal University of Science \& Technology; 2008.

30. Gupta PC, Sreevidya S. Smokeless tobacco use, birth weight and gestational age: population based prospective cohort study of 1217 women in Mumbai, India. BMJ 2004; 328(7455): 1538.

31. Styen K, Wet TD, Saloojee Y, Nel H, Yach D. The influence of maternal cigarette smoking, snuff use and passive smoking on pregnancy outcomes: the birth to ten study. Paeditr Perinatal Epedemiol 2006; 20: 90-9.
32. Wang X, Tager TB, Vunakis HV, Speizer FE, Hanranan JP. Maternal smoking during pregnancy, urine cotinine concentration and birth outcomes: a prospective cohort study. Int $\mathrm{J}$ Epidemiol 1997; 269(5): 978-8.

33. Chowdhury ME, Akhtar S. Effect of Smokeless tobacco consumption on Birth weight among pregnant slum Dwellers in Dhaka city. ICDDR,B. 2003.

34. Hossain M, Saha S. Tobacco and Pregnancy outcomes in a rural area of Bangladesh: APHA Scientific session and Event listing. 3076; 2007 Nov 5- Board 8.

35. Deshmukh JS, Motghare DD, Zodpey SP, Wadhua SK. Low birth weight and associated maternal factors in an urban area. Indian Paediatrics 1998; 35: $33-6$.

36. NABI Biopharmaceuticals. Key Facts about NicVAX and Nicotine Addiction. Available at: http/ www.nabi.com (accessed on :01.04.2009). 